\title{
LILRA6 copy number variation correlates with susceptibility to atopic dermatitis
}

\author{
M. R. López-Álvarez ${ }^{1}$ • W. Jiang ${ }^{2}$ • D. C. Jones ${ }^{1}$ • J. Jayaraman ${ }^{2}$ • C . Johnson ${ }^{2}$ • \\ W. O. Cookson ${ }^{3} \cdot$ M. F. Moffatt ${ }^{3} \cdot$ J. Trowsdale ${ }^{1,2} \cdot$ J. A. Traherne ${ }^{1,2}$
}

Received: 18 February 2016 / Accepted: 10 June 2016 / Published online: 22 June 2016

(C) The Author(s) 2016. This article is published with open access at Springerlink.com

\begin{abstract}
Leukocyte immunoglobulin-like receptors (LILR) are expressed mostly on myelomonocytic cells where they are mediators of immunological tolerance. Two LILR genes, LILRA3 and LILRA6, exhibit marked copy number variation. We assessed the contribution of these genes to atopic dermatitis (AD) by analysing transmission in $378 \mathrm{AD}$ families. The data indicated that copies of LILRA6 were over-transmitted to affected patients. They are consistent with a contribution of LILR genes to AD. They could affect the equilibrium between activating and inhibitory signals in the immune response.
\end{abstract}

Keywords LILR $\cdot$ Atopic dermatitis $\cdot \mathrm{AD} \cdot \mathrm{CNV}$

\section{Introduction}

Atopic dermatitis (AD) is a chronic disease that affects around $10-20 \%$ of children in western countries. It is characterised by pruritus and skin inflammation and is frequently associated with food allergies and asthma (Werfel et al., 2014).

J. A. Traherne

jat51@cam.ac.uk

1 Immunology Division, Department of Pathology, University of Cambridge, Tennis Court Road, Cambridge CB2 1QP, UK

2 Cambridge Institute for Medical Research, University of Cambridge, Cambridge Biomedical Campus, Wellcome Trust/MRC Building, Hills Road, Cambridge CB2 0XY, UK

3 Molecular Genetics and Genomics Section, National Heart and Lung Institute, Imperial College London, Dovehouse Street, London SW3 6LY, UK
There are several factors that contribute to the development of $\mathrm{AD}$ such as environmental conditions (Zutavern et al., 2005; Roduit et al., 2011; Silverberg et al., 2011; Caroline et al., 2012), dysfunction of the skin barrier (Cork et al., 2009; Agrawal and Woodfolk, 2014) and viral and bacterial skin infections (Cho et al., 2001). AD can be developed when there is an abnormal or sustained activation of the skin innate immune system (McGirt and Beck, 2006; Maintz and Novak, 2011; Kasraie and Werfel, 2013, Kuo et al., 2013). Additionally, genetic factors have been associated to the development and vertical transmission of AD (Söderhäll et al., 2007; Irvine et al., 2011). Alterations in the function and signalling pathways and polymorphisms in Toll-like receptor 2 (TLR2) have been associated with the development of $\mathrm{AD}$ (Potaczek et al., 2011; Yu et al., 2015) whilst Killer immunoglobulin-like receptor (KIR), specifically KIR2DS1, seems to be associated with protection from AD (Niepiekło-Miniewska et al., 2013).

Leukocyte immunoglobulin-like receptors (LILR) are also innate immune receptors encoded, like KIR, within the leukocyte receptor complex (LRC) on chromosome 19q13.4 (Barrow and Trowsdale, 2008). LILR display limited genetic diversity (Young et al., 2001) apart from LILRB3 and LILRA6, which exhibit considerable sequence polymorphism in the extracellular domains and LILRA3 and LILRA6 which also show variation in the number of copies (Torkar et al., 2000; Sudmant et al., 2010; Bashirova et al., 2014; Lopez-Alvarez et al., 2014). LILR can regulate the activity of TLR (Brown et al. 2004, Cao et al., 2009) and mediate immunological tolerance (Manavalan et al., 2003; Kim-Schulze et al., 2006; Anderson and Allen, 2009). Currently, there is no data about the role of LILRB3 and LILRA6, although their recently discovered interaction with a cytokeratin 8-associated ligand suggests they could shape local inflammatory responses to epithelial tumours (Jones et al., 2016). LILRA3 has been described as a 
soluble receptor that binds HLA class I molecules and it has been associated with multiple sclerosis (An et al., 2016), systemic lupus erythematosus and Sjögren's syndrome (Du et al., 2014).

Despite the strong genetic component together with the implication of the innate immune response in $\mathrm{AD}$, in particular TLR and KIR receptors, there is no data available considering the association of LILR genes with AD. This is a preliminary study to assess the impact of the variability of LILRA3 and LILRA6 loci among members of $378 \mathrm{AD}$ families.

DNA samples, obtained from the UK DNA Banking Network (DBN), consisted of 1482 Caucasoid individuals from 378 families. Three hundred fifty-eight children had active AD. The childrens' mean age was 10 years ( \pm 4 years). One hundred ninety-six (55\%) were male subjects (Sandford et al., 1995; Morar et al., 2006). Children lacking signs of disease were classified as unaffected. No significant association between sex or age and development of $\mathrm{AD}$ was found $(p>0.05)$. All subjects were of initial European ancestry and gave their informed consent.

LILRA3 and LILRA6 copy number was determined by quantitative PCR on genomic DNA extracted using standard techniques. Forward and reverse primers and a dual-labelled probe were designed to specifically amplify LILRA3 and LILRA6 genes avoiding any allelic variation identified to date (Lopez-Alvarez et al., 2014). Sequences were analysed for specificity using the primerBLAST tool from the National Centre for Biotechnology Information (http://www.ncbi.nlm. nih.gov/tools/primer-blast). In addition, all reactions contained specific primers and a probe for the STAT6 gene, which has two copies per diploid human genome, and was used as an endogenous reference gene. All reactions were performed in quadruplicate for each sample to increase the accuracy of copy number scoring. Positive controls for each copy number type from IHW cell lines (Lopez-Alvarez et al., 2014) and a negative non-template control were included on each microtiter plate.
A total of $10 \mathrm{ng}$ of genomic DNA was amplified under the following PCR conditions: $5 \mathrm{~min}$ at $95{ }^{\circ} \mathrm{C}$, followed by 40 cycles of $95{ }^{\circ} \mathrm{C}$ for $15 \mathrm{~s}$ and $66^{\circ} \mathrm{C}$ for $50 \mathrm{~s}$, followed by $10 \mathrm{~s}$ at $40{ }^{\circ} \mathrm{C}$, using the LightCycler 480 System (Roche Diagnostics Ltd., Burgess Hill, UK). LILR copy number was determined by a quantitative PCR comparative $\mathrm{Ct}$ method (Schmittgen and Livak, 2008).

Family-based association analysis was carried out using the transmission disequilibrium test (TDT) to examine the transmission rates for the number of copies of LILRA3 and LILRA6 from parents to affected offspring. The analysis was performed using Unphased software (Dudbridge, 2008) which implements maximum-likelihood inference on genotype effects. In addition, FBAT software version V2.04 was used to perform the association analyses using the additive model and bi-allelic mode (data not shown).

First of all, we determined the variation in the number of copies of LILRA3 and LILRA6 loci in the cohort of families. The number of copies of LILRA3 per haplotype varied from 0 to 2 copies, whilst LILRA6 showed between 0 and 4 copies. One copy was the most frequent for LILRA3 and LILRA6 with frequencies over $70 \%$ in both cases (Table 1). The frequencies obtained per diploid genome matched very closely to previously reported (data not shown, Lopez-Alvarez et al., 2014).

Regarding the transmission of LILRA3 and LILRA6 among families, in relation to disease (Table 2), LILRA6 showed a significant association with $\mathrm{AD}(p=0.0025)$. There was a slight over-transmission of one copy of LILRA6 to affected children (0.7197 vs. 0.6842 , $p=0.002$, OR $1.45,95 \%$ CI 1.14-1.85), suggesting that the presence of one copy of LILRA6 on each chromosome could be a risk factor for AD. In contrast, haplotypes missing LILRA6 (0 copies per haplotype) were undertransmitted (0.0636 vs. 0.0933$)$. Analysis of the data with FBAT software (see Methods) also revealed the above associations (data not shown).
Table 1 Frequencies of the number of copies in LILRA3 and LILRA6 per haplotype

\begin{tabular}{llcccc}
\hline Marker & Number of copies & Total & AD & & \multirow{2}{*}{$P$ value } \\
\cline { 3 - 5 } & & & Unaffected & Affected & \\
\hline LILRA3 & 0 & $613(20.7)$ & $401(20.9)$ & $212(20.3)$ & 0.714 \\
& 1 & $2339(78.9)$ & $1512(78.7)$ & $827(79.4)$ & \\
LILRA6 & 2 & $12(0.4)$ & $9(0.5)$ & $3(0.1)$ & \\
& 0 & $217(7.3)$ & $143(7.4)$ & $74(7.1)$ & 0.948 \\
& 1 & $2107(71.2)$ & $1370(71.3)$ & $737(70.7)$ & \\
& 2 & $617(20.8)$ & $395(20.6)$ & $222(21.3)$ & \\
& 3 & $19(0.6)$ & $12(0.6)$ & $7(0.7)$ & \\
\hline
\end{tabular}

Number of copies denotes copies per haplotype. Frequencies are given in the parentheses 
Table 2 TDT results for LILRA3 and LILRA6 in AD affected families

\begin{tabular}{rlrrlll}
\hline & Number of copies & Trans & Untrans & T-Freq & U-Freq & $P$ value \\
\hline LILRA3 & 0 & 157 & 432 & 0.2224 & 0.2006 & 0.980518 \\
& 1 & 547 & 1715 & 0.7748 & 0.7962 & \\
LILRA6 & 2 & 2 & 7 & 0.002833 & 0.00325 & \\
& 0 & 44 & 200 & 0.06358 & 0.09325 & 0.00254137 \\
& 1 & 498 & 1467 & 0.7197 & 0.6842 & \\
& 2 & 145 & 462 & 0.2095 & 0.20155 & \\
& 3 & 4 & 14 & 0.00578 & 0.00653 & \\
& 4 & 1 & 1 & 0.001445 & 0.0004664 & \\
\hline
\end{tabular}

Number of copies denotes copies of the gene on the same haplotype. Trans is the estimated count of this haplotype to affected offspring. Untrans is the estimated number of non-transmissions of this haplotype. TDT-like counts of transmitted and untransmitted allelic copies are not given as they cannot be accurately defined when parents are missing. T-Freq is the frequency of the haplotype in affected offspring. U-Freq is the frequency of the haplotype among untransmitted haplotypes. $P$ value is the overall significance for the locus
In several families, the transmission of LILRA3 and LILRA6 genes was consistent with duplication of these genes on a chromosome. Twelve individuals $(\sim 1 \%)$, distributed among five families, would have two copies of LILRA3 on the same chromosome, being the only possible arrangement to explain the segregation through the next generation. The incidence of two copies of LILRA6 on the same chromosome was more frequent $(\sim 7 \%)$. In those individuals with a higher number of copies of LILRA6, the number of copies was not evenly distributed on each chromosome. For example, some possessed four copies on one chromosome and two on the other.

This is the first study considering the effect of LILR receptors on the development of $A D$. We analysed the variation in the number of copies of LILRA3 and LILRA6 genes in a series of a family trios suffering from the disease.

$\mathrm{AD}$ is characterised by a Th2-mediated response (Novak et al., 2003; Chu et al., 2011) triggered by dendritic cells (DC) (Moser and Murphy, 2000). LILRA3 is a soluble protein which seems to be an antagonist of inhibitory LILRs (Poon et al., 2005) and could also have an important role in establishing Th1 or Th2 responses (Thomas et al., 2010). The lack of LILRA3 could lead to the early conclusion of the antiviral defence, supporting chronic infection and favouring the development of autoimmune diseases, like multiple sclerosis and Sjögren's syndrome (Pender, 2009; Thomas et al., 2010). Unlike in autoimmune diseases (Wiśniewski et al., 2013; Du et al., 2014), the number of copies of LILRA3 was not significantly different between $\mathrm{AD}$ unaffected and affected individuals without considering their relatedness (Table 1) or in transmission frequencies of gene copies between unaffected and affected offspring.

LILRA6 is an activating receptor encoded by a polymorphic gene that shows variation in the number of copies (Bashirova et al., 2014; Lopez-Alvarez et al., 2014). Like LILRA3, there were no differences in the number of copies between unaffected and AD affected individuals but we observed significant differences in the transmission of the number of copies of LILRA6 within families (Table 2). The results showed a modest over-transmission of one copy of LILRA6 whilst haplotypes lacking this gene were under transmitted. These results suggest that the lack of LILRA6 could be a protective factor against AD. Indeed, the activating nature of this receptor upon binding with the specific ligand could trigger the immune response and promote the development of atopic dermatitis. In contrast, two or more copies of LILRA6 showed no influence on disease risk. The reason for this is unclear at present but it could mean that the paralogs are disrupted genes or that they differ functionally.

LILRA6 CNV may influence the level of the activating receptor on the cell surface, potentially affecting signalling upon LILRB3/A6 ligation (Bashirova et al., 2014). Thus, the variation observed here could affect the equilibrium between activating and inhibitory signals and the balance of the immune response. An alternative explanation could be that the effects we observe reflect linked genes such as KIR on NK cells. In this context, some HLA class I alleles, such as HLAB*57:01 and HLA-B*44:02 which are ligands for KIR receptors, have been associated with AD in several GWAS studies (Hirota et al., 2012; Weidinger et al., 2013). Also, KIR2DS1 may be a protective factor in $\mathrm{AD}$, although its effect remains unclear (Kusnierczyk, 2013).

In conclusion, we have assessed the variation in the number of copies of LILRA3 and LILRA6 in a series of AD family trios. Among them, only the transmission of one copy of LILRA6 within families was potentially related to the development of AD. Further studies should be done in order to clarify the role of LILRA6 and its possible ligands in the development of AD. 
Acknowledgments This work was funded by the Medical Research Council (MRC) and Association for International Cancer Research (AICR) with additional support from the Wellcome Trust and the National Institute for Health Research (NIHR) Cambridge Biomedical Research Centre. María Rocío López-Álvarez was funded by Ministerio de Educación of Spain, under the programme "Programa Nacional de Movilidad de Recursos Humanos del Plan Nacional de I-D+i 2008-2011" and Fundación Séneca 04087/GERM/06 Project.

\section{Compliance with ethical standards}

Conflict of interest The authors declare they have no conflict of interest.

Open Access This article is distributed under the terms of the Creative Commons Attribution 4.0 International License (http:// creativecommons.org/licenses/by/4.0/), which permits unrestricted use, distribution, and reproduction in any medium, provided you give appropriate credit to the original author(s) and the source, provide a link to the Creative Commons license, and indicate if changes were made.

\section{References}

Agrawal R, Woodfolk JA (2014) Skin barrier defects in atopic dermatitis. Curr Allergy Asthma Rep 14(5):433

An H, Lim C, Guillemin GJ, et al. (2016) Serum leukocyte immunoglobulin-like receptor A3 (LILRA3) is increased in patients with multiple sclerosis and is a strong independent indicator of disease severity; $6.7 \mathrm{kbp}$ LILRA3 gene deletion is not associated with diseases susceptibility. PLoS One 11(2):e0149200

Anderson KJ, Allen RL (2009) Regulation of T cell immunity by leukocyte immunoglobulin-like receptors: innate immune receptors for self antigen-presenting cells. Immunology 127(1):8-17

Barrow AD, Trowsdale J (2008) The extended human leukocyte receptor complex: diverse ways of modulating immune responses. Immunol Rev 224:98-123

Bashirova AA, Apps R, Vince N, et al. (2014) Diversity of the human LILRB3/A6 locus encoding a myeloid inhibitory and activating receptor pair. Immunogenetics 66(1):1-8

Brown D, Trowsdale J, Allen R (2004) The LILR family: modulators of innate and adaptive immune pathways in health and disease. Tissue Antigens 64(3):215-225

Cao W, Bover L, Cho M, et al. (2009) Regulation of TLR7/9 responses in plasmacytoid dendritic cells by BST2 and ILT7 receptor interaction. J Exp Med 206(7):1603-1614

Caroline R, Frei R, Loss G, et al. (2012) Development of atopic dermatitis according to age of onset and association with early-life exposures. J Allergy Clin Immunol 130:130-136

Cho SH, Strickland I, Boguniewicz M, et al. (2001) Fibronectin and fibrinogen contribute to the enhanced binding of Staphylococcus aureus to atopic skin. J Allergy Clin Immunol 108:269-274

Chu CC, Di Meglio P, Nestle FO (2011) Harnessing dendritic cells in inflammatory skin diseases. Semin Immunol 23(1):28-41

Cork MJ, Danby SG, Vasilopoulos Y, et al. (2009) Epidermal barrier dysfunction in atopic dermatitis. J Investig Dermatol 129(8):1892-1908

Du Y, Su Y, He J, et al. (2014) Impact of the leukocyte immunoglobulinlike receptor A3 (LILRA3) on susceptibility and subphenotypes of systemic lupus erythematosus and Sjögren's syndrome. Ann Rheum Dis. doi:10.1136/annrheumdis-2013-204441
Dudbridge F (2008) Likelihood-based association analysis for nuclear families and unrelated subjects with missing genotype data. Hum Hered 66(2):87-98

Hirota T, Atsushi T, Kubo M, et al. (2012) Genome-wide association study identifies eight new susceptibility loci for atopic dermatitis in the Japanese population. Nat Genet 44:1222-1226

Irvine AD, McLean WH, Leung DY (2011) Filaggrin mutations associated with skin and allergic diseases. $\mathrm{N}$ Engl J Med 365: $1315-1327$

Jones DC, Hewitt CR, López-Álvarez MR, et al. (2016) Allele-specific recognition by LILRB3 and LILRA6 of a cytokeratin 8-associated ligand on necrotic glandular epithelial cells. Oncotarget. doi:10.18632/oncotarget.6905

Kasraie S, Werfel T (2013) Role of macrophages in the pathogenesis of atopic dermatitis. Mediat Inflamm. Article ID 942375

Kim-Schulze S, Scotto L, Vlad G, et al. (2006) Recombinant Ig-like transcript 3-Fc modulates $\mathrm{T}$ cell responses via induction of Th anergy and differentiation of $\mathrm{CD} 8^{+} \mathrm{T}$ suppressor cells. J Immunol 176(5):2790-2798

Kuo IH, Yoshida T, De Benedetto A, et al. (2013) The cutaneous innate immune response in patients with atopic dermatitis. J Allergy Clin Immunol 131(2):266-278

Kusnierczyk P (2013) Killer cell immunoglobulin-like receptor gene associations with autoimmune and allergic diseases, recurrent spontaneous abortion, and neoplasms. Front Immunol. doi:10.3389 /fimmu.2013.00008

Lopez-Alvarez MR, Jones DC, Jiang W, et al. (2014) Copy number and nucleotide variation of the LILR family of myelomonocytic cell activating and inhibitory receptors. Immunogenetics 66(2):73-83

Maintz L, Novak N (2011) Modifications of the innate immune system in atopic dermatitis. J Innate Immun 3(2):131-141

Manavalan JS, Rossi PC, Vlad G, et al. (2003) High expression of ILT3 and ILT4 is a general feature of tolerogenic dendritic cells. Transpl Immunol 11(3-4):245-258

McGirt LY, Beck LA (2006) Innate immune defects in atopic dermatitis. J Allergy Clin Immunol 118:202-208

Morar N, Bowcock AM, Harper JI, et al. (2006) Investigation of the chromosome 17q25 PSORS2 locus in atopic dermatitis. J Investig Dermatol 126(3):603-606

Moser M, Murphy KM (2000) Dendritic cell regulation of $\mathrm{T}_{\mathrm{H}} 1-\mathrm{T}_{\mathrm{H}} 2$ development. Nat Immunol 1:199-205

Niepiekło-Miniewska W, Majorczyk E, Matusiak L, et al. (2013) Protective effect of the KIR2DS1 gene in atopic dermatitis. Gene 527(2):594-600

Novak N, Bieber T, Leung DYM (2003) Immune mechanisms leading to atopic dermatitis. J Allergy Clin Immunol 112:S128-S139

Pender MP (2009) Preventing and curing multiple sclerosis by controlling Epstein-Barr virus infection. Autoimmun Rev 8(7):563-568

Poon K, Montamat-Sicotte D, Cumberbatch N, et al. (2005) Expression of leukocyte immunoglobulin-like receptors and natural killer receptors on virus-specific $\mathrm{CD}^{+} \mathrm{T}$ cells during the evolution of EpsteinBarr virus-specific immune responses in vivo. Viral Immunol 18: $513-522$

Potaczek DP, Nastalek M, Okumura K, et al. (2011) An association of TLR2-16934A > T polymorphism and severity/phenotype of atopic dermatitis. J Eur Acad Dermatol Venereol 25:715-721

Roduit C, Wohlgensinger J, Frei R, et al. (2011) Prenatal animal contact and gene expression of innate immunity receptors at birth are associated with atopic dermatitis. J Allergy Clin Immunol 127: 179-185

Sandford AJ, Moffatt MF, Daniels SE, et al. (1995) A genetic map of chromosome 11q, including the atopy locus. Eur J Hum Genet 3(3):188-194

Schmittgen TD, Livak KJ (2008) Analyzing real-time PCR data by the comparative C(T) method. Nat Protoc 3:1101-1108 
Silverberg JI, Kleiman E, Lev-Tov H, et al. (2011) Association between obesity and atopic dermatitis in childhood: a case control study. J Allergy Clin Immunol 127:1180-1186

Söderhäll C, Marenholz I, Kerscher T, et al. (2007) Variants in a novel epidermal collagen gene (COL29A1) are associated with atopic dermatitis. PLoS Biol 5(9):e242

Sudmant PH, Kitzman JO, Antonacci F, et al. (2010) Diversity of human copy number variation and multicopy genes. Science 330(6004):641-646

Thomas R, Matthias T, Witte T (2010) Leukocyte immunoglobulin-like receptors as new players in autoimmunity. Clin Rev Allergy Immunol 38:159-162

Torkar M, Haude A, Milne S, et al. (2000) Arrangement of the ILT gene cluster: a common null allele of the ILT6 gene results from a $6.7-\mathrm{kbp}$ deletion. Eur J Immunol 30:3655-3662

Weidinger S, Willis-Owen SAG, Kamatani Y, et al. (2013) A genome-wide association study of atopic dermatitis identifies loci with overlapping effects on asthma and psoriasis. Hum Mol Genet 22(23):4841-4856
Werfel T, Schwerk N, Hansen G, et al. (2014) The diagnosis and graded therapy of atopic dermatitis. Dtsch Arztebl Int 111(29-30):509-520

Wiśniewski A, Wagner M, Nowak I, et al (2013) 6.7-kbp deletion in LILRA3 (ILT6) gene is associated with later onset of the multiple sclerosis in a Polish population. Hum Immunol 74(3):353-357

Young NT, Canavez F, Uhrberg M, et al. (2001) Conserved organization of the ILT/LIR gene family within the polymorphic human leukocyte receptor complex. Immunogenetics 53(4): 270-278

Yu Y, Zhang Y, Zhang J, et al. (2015) Impaired Toll-like receptor-2-mediated Th1 and Th17/22 cytokines secretion in human peripheral blood mononuclear cells from patients with atopic dermatitis. Transl Med 13:384

Zutavern A, Hirsch T, Leupold W, et al. (2005) Atopic dermatitis, extrinsic atopic dermatitis and the hygiene hypothesis: results from a cross-sectional study. Clin Exp Allergy 35: $1301-1308$ 